\title{
The Equivariant Toda Lattice
}

By

\section{Ezra GetzleR*}

The Toda lattice is an infinite dimensional dynamical system of commuting flows

$$
\left(\partial, \delta_{n}, \bar{\delta}_{n} \mid n>0\right)
$$

acting on functions $\left(q, a_{k}, \bar{a}_{k} \mid k>0\right)$ defined on a one-dimensional lattice. In the limit of small lattice spacing $\varepsilon$, which is all that will concern us here (Takasaki and Takebe [12]), the functions $\left(q, a_{k}, \bar{a}_{k}\right)$ become functions of a real parameter $x$, and the role of translation by one unit of the lattice is taken by the operator $\mathrm{E}=e^{\varepsilon \partial}$, where $\partial$ is the infinitesimal generator of translations in $x$.

The derivations $\delta_{1}$ and $\bar{\delta}_{1}$ act on the variables $q, a_{1}$ and $\bar{a}_{1}$ by the formulas

$$
\delta_{1} \bar{a}_{1}=\bar{\delta}_{1} a_{1}=\nabla q, \quad \delta_{1} \log q=\nabla a_{1}, \quad \bar{\delta}_{1} \log q=\nabla \bar{a}_{1},
$$

where $\nabla: \mathcal{A} \rightarrow \mathcal{A}$ is the infinite-order differential operator

$$
\begin{aligned}
\nabla & =\varepsilon^{-1}\left(\mathrm{E}^{1 / 2}-\mathrm{E}^{-1 / 2}\right)=\sum_{k=0}^{\infty} \frac{\varepsilon^{2 k} \partial^{2 k+1}}{2^{2 k}(2 k+1) !} \\
& =\partial+\frac{1}{24} \varepsilon^{2} \partial^{3}+O\left(\varepsilon^{4}\right) .
\end{aligned}
$$

These formulas imply the Toda equation:

$$
\delta_{1} \bar{\delta}_{1} \log q=\nabla^{2} q .
$$

The higher Toda flows are symmetries of this equation.

An abstract mathematical formulation of the Toda lattice is obtained by realizing the derivations $\left(\partial, \delta_{n}, \bar{\delta}_{n}\right)$ on the free differential algebra $\mathcal{A}=$

Communicated by K. Saito. Received September 18, 2002. Revised May 6, 2003.

2000 Mathematics Subject Classification(s): 37K10, 53D45.

The author was partially supported by the NSF under grant DMS-0072508, and, through the Institute for Advanced Study, under grant DMS-9729992.

* Department of Mathematics, Northwestern University, Evanston, Illinois, 60208 USA. e-mail: getzler@northwestern.edu 
$\mathbb{Q}_{\varepsilon}\left\{q, a_{k}, \bar{a}_{k} \mid k>0\right\}$, defined over the ring $\mathbb{Q}_{\varepsilon}=\mathbb{Q} \llbracket \varepsilon \rrbracket$. Reductions of the Toda lattice correspond to differential ideals $\mathcal{I}$ in $\mathcal{A}$ invariant under conjugation and closed under the derivations $\delta_{n}$ and $\bar{\delta}_{n}$. For example, the Toda chain is described by the differential ideal with generators

$$
\mathcal{I}=\left\{a_{1}-\bar{a}_{1}, a_{2}-q, \bar{a}_{2}-q, a_{k}, \bar{a}_{k} \mid k>2\right\},
$$

so that $\mathcal{A} / \mathcal{I} \cong \mathbb{Q}_{\varepsilon}\{q, v\}$, where $v$ is the image of $a_{1} \in \mathcal{A}$.

In this paper, we study a new reduction of the Toda lattice, which we call the equivariant Toda lattice. If $\nu$ is a formal parameter, this reduction is defined by the following constraint on the Lax operator:

$$
\left(\delta_{1}-\bar{\delta}_{1}\right) L=\nu \partial L
$$

Let $\mathcal{I}_{\nu} \subset \mathcal{A}[\nu]$ be the corresponding differential ideal. We prove that the differential algebra $\mathcal{A}[\nu] / \mathcal{I}_{\nu}$ is isomorphic to

$$
\mathbb{Q}_{\varepsilon, \nu}\{q, v, \bar{v}\} /(\nu \partial q-\nabla(v-\bar{v})) \otimes_{\mathbb{Q}_{\varepsilon, \nu}} \mathbb{Q}_{\varepsilon, \nu}\left[z_{k}, \bar{z}_{k} \mid k>0\right],
$$

where $\mathbb{Q}_{\varepsilon, \nu}=\mathbb{Q}_{\varepsilon}[\nu], v$ and $\bar{v}$ are the images of $a_{1}$ and $\bar{a}_{1} \in \mathcal{A}$, and $z_{k}$ and $\bar{z}_{k}$ are constants of motion, which may be defined by the following equation:

$$
\left(L-\nu+\sum_{k=1}^{\infty} z_{k} L^{-k}\right) \frac{\partial L}{\partial v}=L .
$$

In an influential paper, Eguchi and Yang [3] conjectured that the Gromov-Witten invariants of $\mathbb{C P}^{1}$ are related to the Toda chain. (For more on this conjecture, see Eguchi, Hori and Yang [4], Pandharipande [10] and Getzler [5].) The equivariant Toda conjecture, as formulated in this paper in terms of the equivariant Toda lattice, provides a similar description of the equivariant Gromov-Witten invariants of $\mathbb{C P}^{1}$, which specializes to the conjecture of Eguchi and Yang in the non-equivariant limit.

Let $\mathbb{T}$ be the multiplicative group of $\mathbb{C}$, and let $X$ be a topological space with an action of $\mathbb{T}$. The equivariant cohomology $H_{\mathbb{T}}^{*}(X, \mathbb{Z})$ of $X$ is a module over the graded ring $H_{\mathbb{T}}^{\bullet}=H^{\bullet}(B \mathbb{T}, \mathbb{Z}) \cong \mathbb{Z}[\nu]$, where $\nu \in H_{\mathbb{T}}^{2}$. The equivariant cohomology $H_{\mathbb{T}}^{\bullet}\left(\mathbb{C} \mathbb{P}^{1}, \mathbb{Z}\right)$ of the projective line $\mathbb{C P}^{1}$ admits a presentation

$$
H_{\mathbb{T}}^{\bullet}\left(\mathbb{C P}^{1}, \mathbb{Z}\right) \cong \mathbb{Z}[H, \nu] /(H(H-\nu)),
$$

where $H$ is the equivariant Chern class $c_{1}(\mathcal{O}(1)) \in H_{\mathbb{T}}^{2}\left(\mathbb{C P}^{1}, \mathbb{Z}\right)$. 
Denote the $k$ th descendants of the cohomology classes 1 and $H$ in GromovWitten theory by $\tau_{k, P}$ and $\tau_{k, Q}$ respectively; also, abbreviate $\tau_{0, P}$ and $\tau_{0, Q}$ to $P$ and $Q$. The genus 0 equivariant Gromov-Witten invariants of $\mathbb{C P}^{1}$ are integrals over the moduli space $\overline{\mathcal{M}}_{0, n}\left(\mathbb{C P}^{1}\right)$ of stable maps of genus $g$ with $n$ marked points:

$$
\left\langle\tau_{k_{1}, P} \ldots \tau_{k_{m}, P} \tau_{\ell_{1}, Q} \ldots \tau_{\ell_{n}, Q}\right\rangle_{g} \in H_{\mathbb{T}}^{\bullet} \otimes \mathbb{Q} \cong \mathbb{Q}[\nu]
$$

The large phase space is the formal affine space with coordinates $\left\{s_{k}, t_{k} \mid k \geq\right.$ $0\}$. The genus $g$ Gromov-Witten potential $\mathcal{F}_{g}$ of $\mathbb{C P}^{1}$ is the generating function on the large phase space given by the formula

$$
\mathcal{F}_{g}=\sum_{m, n=0}^{\infty} \frac{1}{m ! n !} \sum_{\substack{k_{1}, \ldots, k_{m} \\ \ell_{1}, \ldots, \ell_{n}}} s_{k_{1}} \ldots s_{k_{m}} t_{\ell_{1}} \ldots t_{\ell_{n}}\left\langle\tau_{k_{1}, P} \ldots \tau_{k_{m}, P} \tau_{\ell_{1}, Q} \ldots \tau_{\ell_{n}, Q}\right\rangle_{g}
$$

We may combine the Gromov-Witten potentials into a single generating function by interpreting $\varepsilon$ as a genus expansion parameter, and writing

$$
\mathcal{F}=\sum_{g=0}^{\infty} \varepsilon^{2 g} \mathcal{F}_{g}
$$

Based on explicit calculations using the topological recursion relations in genus 0 and 1, Pandharipande conjectured [11] that the following equation holds for the total Gromov-Witten potential:

$$
\partial_{0} \bar{\partial}_{0} \mathcal{F}=\exp \left(\nabla^{2} \mathcal{F}\right)
$$

Here, $\nabla=\varepsilon^{-1}\left(\mathrm{E}^{1 / 2}-\mathrm{E}^{-1 / 2}\right)$, where $\mathrm{E}=e^{\varepsilon \partial}$, and $\partial=\partial / \partial s_{0}$.

On applying the operator $\nabla^{2}$ to both sides of (4) and identifying the vector fields $\partial_{0}$ and $\bar{\partial}_{0}$ with the Toda flows $\delta_{1}$ and $\bar{\delta}_{1}$, we obtain the Toda equation (2) for $q=\exp \left(\nabla^{2} \mathcal{F}\right)$. Observe that $\partial_{1}-\bar{\partial}_{1}=\nu \partial$; this equation is formally identical to the constraint defining the equivariant Toda lattice.

The equivariant Toda lattice and the equivariant Gromov-Witten theory of $\mathbb{C P}^{1}$ each involve sequences $\left\{\delta_{n}, \bar{\delta}_{n}\right\}$ and $\left\{\partial_{n}=\partial / \partial t_{n}, \bar{\partial}_{n}=\partial / \partial t_{n}-\nu \partial / \partial s_{n}\right\}$ of commuting derivations, in the first case on the algebra $\mathcal{A}[\nu] / \mathcal{I}_{\nu}$, and in the second case on functions on the large phase space. These sequences of vector fields may be compared by means of a morphism

$$
\mathcal{A}[\nu] / \mathcal{I}_{\nu} \longrightarrow \mathbb{Q}_{\varepsilon, \nu} \llbracket s_{k}, t_{k} \mid k \geq 0 \rrbracket
$$


of differential algebras which sends the generators $q, v$ and $\bar{v}$ to $\exp \left(\nabla^{2} \mathcal{F}\right)$, $\nabla \partial_{0} \mathcal{F}$ and $\nabla \bar{\partial}_{0} \mathcal{F}$, and the constants $z_{k}$ and $\bar{z}_{k}$ to 0 . In fact, the following relationship between these flows holds:

$$
\begin{aligned}
\sum_{k=0}^{\infty} z^{k+1} \partial_{k} & =\sum_{n=1}^{\infty} \frac{z^{n} \delta_{n}}{(1+z \nu)(2+z \nu) \ldots(n+z \nu)} \\
\sum_{k=0}^{\infty} z^{k+1} \bar{\partial}_{k} & =\sum_{n=1}^{\infty} \frac{z^{n} \bar{\delta}_{n}}{(1-z \nu)(2-z \nu) \ldots(n-z \nu)}
\end{aligned}
$$

We conjectured this in a preprint of this paper, based on a proof of the result in genus 0 (see Section 4), together with calculations in genus 1 for small values of $n$; it has recently been proved by Okounkov and Pandharipande [9], along with the equivariant Toda equation (4). Thus, the equivariant Toda lattice yields a description of the equivariant Gromov-Witten invariants of $\mathbb{C P}^{1}$ in terms of a Lax operator whose coefficients are obtained by an explicit recursion. In particular, the descendent flows $\partial / \partial s_{k}$ of the puncture operator $P$ are given in the non-equivariant limit by the formula

$$
\frac{\partial}{\partial s_{k}}=\lim _{\nu \rightarrow 0}\left(\frac{\delta_{k+1}-\bar{\delta}_{k+1}}{\nu(k+1) !}-\frac{c_{k}\left(\delta_{k}+\bar{\delta}_{k}\right)}{k !}\right)
$$

where $c_{k}$ is the harmonic number $c_{k}=1+\frac{1}{2}+\cdots+\frac{1}{k}$; this is exactly as conjectured by Eguchi and Yang [3].

In the second part of this paper (Sections 5-8), we relate the equivariant Toda lattice to the dressing operator formalism. Let $\log (L)=W \log (\Lambda) W^{-1}$ be the logarithm of the Lax operator $L$, related to the operator $\ell=\varepsilon(\partial W) W^{-1}$ by the formula

$$
\log (L)=\log (\Lambda)-\ell
$$

Borrowing ideas of Carlet, Dubrovin and Zhang [1], we show that the equivariant Toda lattice may be characterized by the expansion

$$
\Lambda+v+q \Lambda^{-1}=L+\nu \ell-\sum_{k=1}^{\infty} \frac{z_{k}}{k} L^{-k} .
$$

In particular, the equation $\left(\delta_{1}-\bar{\delta}_{1}\right) W=\nu \partial W$ is equivalent to the vanishing of the coefficients $z_{k}$. We also show that the equivariant Toda lattice has a Hamiltonian structure which is a deformation of the first Hamiltonian structure of the Toda chain. (We were however unable to find a bihamiltonian structure.) This gives a more direct relationship between the results of Okounkov and Pandharipande [9] and the original Toda conjecture. 
This paper closes with an appendix in which the formulas (5a) and (5b) relating $\left\{\partial_{k}, \bar{\partial}_{k}\right\}$ and $\left\{\delta_{n}, \bar{\delta}_{n}\right\}$ are inverted.

\section{$\S 1$ Difference Operators}

In this section, we recall the mathematical structure underlying the Toda lattice; this material is adapted from the fundamental papers of Ueno and Takasaki [13] and Kupershmidt [8].

All of the commutative algebras which we consider in this paper carry an involution $p \mapsto \bar{p}$, and all ideals which we consider are closed under this involution. By a differential algebra, we mean a commutative algebra with derivation $\partial$ such that

$$
\partial \bar{p}=\overline{\partial p}
$$

A differential ideal is an ideal closed under the action of the differential $\partial$. If $S$ is a subset of a differential algebra $\mathcal{A}$, denote the differential ideal generated by $S \cup \bar{S}$ by $(S)$, where $\bar{S}=\{\bar{x} \mid x \in S\}$ is the conjugate of $S$.

If $\mathcal{A}$ is a differential algebra and $S$ is a set, the free differential algebra $\mathcal{A}\{S\}$ generated by $S$ is the polynomial algebra

$$
\mathcal{A}\left[\partial^{n} x, \partial^{n} \bar{x} \mid x \in S, n \geq 0\right],
$$

with differential $\partial\left(\partial^{n} x\right)=\partial^{n+1} x$.

An evolutionary derivation $\delta$ of a differential algebra $\mathcal{A}$ is a derivation such that $[\partial, \delta]=0$. The evolutionary derivations form a Lie subalgebra of the Lie algebra of derivations of $\mathcal{A}$, with involution

$$
\bar{\delta} p=\overline{\delta \bar{p}}
$$

Let $\mathcal{A}$ be a differential algebra over $\mathbb{Q}_{\varepsilon}$, and let $q \in \mathcal{A}$ be a regular element (that is, having no zero-divisors) such that $\bar{q}=q$. The localization $q^{-1} \mathcal{A}$ of $\mathcal{A}$ is a filtered differential algebra, with differential $\partial\left(q^{-1}\right)=-q^{-2} \partial q$. Let $\Phi_{ \pm}(\mathcal{A}, q)$ be the associative algebras of difference operators

$$
\begin{aligned}
& \Phi_{+}(\mathcal{A}, q)=\left\{\sum_{k=-\infty}^{\infty} p_{k} \Lambda^{k} \mid p_{k} \in q^{-k} \mathcal{A}, p_{k}=0 \text { for } k \ll 0\right\}, \\
& \Phi_{-}(\mathcal{A}, q)=\left\{\sum_{k=-\infty}^{\infty} p_{k} \Lambda^{k} \mid p_{k} \in \mathcal{A}, p_{k}=0 \text { for } k \gg 0\right\},
\end{aligned}
$$


with product

$$
\sum_{i} a_{i} \Lambda^{i} \cdot \sum_{j} b_{j} \Lambda^{j}=\sum_{k}\left(\sum_{i+j=k}\left(\mathrm{E}^{-j / 2} a_{i}\right)\left(\mathrm{E}^{i / 2} b_{j}\right)\right) \Lambda^{k} .
$$

Note that $\Phi_{-}(\mathcal{A}, q)$ is in fact independent of $q$.

Let $A \mapsto A_{ \pm}$be the projections on $\Phi_{ \pm}(\mathcal{A}, q)$ defined by the formulas

$$
\left(\sum_{k=-\infty}^{\infty} p_{k} \Lambda^{k}\right)_{+}=\sum_{k=0}^{\infty} p_{k} \Lambda^{k},\left(\sum_{k=-\infty}^{\infty} p_{k} \Lambda^{k}\right)_{-}=\sum_{k=-\infty}^{-1} p_{k} \Lambda^{k}
$$

We see that $A=A_{-}+A_{+}$. Define the residue res : $\Phi_{ \pm}(\mathcal{A}, q) \rightarrow \mathcal{A}$ by the formula

$$
\operatorname{res}\left(\sum_{k=-\infty}^{\infty} p_{k} \Lambda^{k}\right)=p_{0}
$$

For $k \in \mathbb{Z}$, let $[k]$ be the isomorphism of $\mathcal{A}$

$$
[k]=\frac{\mathrm{E}^{k / 2}-\mathrm{E}^{-k / 2}}{\mathrm{E}^{1 / 2}-\mathrm{E}^{-1 / 2}}=\sum_{j=1}^{k} \mathrm{E}^{(k+1) / 2-j}=k+O\left(\varepsilon^{2}\right) .
$$

Define $q^{[k]}$ by the recursion

$$
q^{[k+1]}=\mathrm{E}^{k} q \cdot \mathrm{E}^{-1 / 2} q^{[k]},
$$

with initial condition $q^{[0]}=1$. The involution

$$
A=\sum_{k=-\infty}^{\infty} p_{k} \Lambda^{k} \mapsto \bar{A}=\sum_{k=1}^{\infty} \bar{p}_{k} q^{[k]} \Lambda^{-k}+\bar{p}_{0}+\sum_{k=1}^{\infty} \bar{p}_{-k} q^{-[k]} \Lambda^{k},
$$

defines an anti-isomorphism between the algebras $\Phi_{+}(\mathcal{A}, q)$ and $\Phi_{-}(\mathcal{A}, q)$.

\section{$\S 2 . \quad$ The Toda Lattice}

To formulate the Toda lattice, we introduce the differential algebra

$$
\mathcal{A}=\mathbb{Q}_{\varepsilon}\left\{q, a_{k} \mid k>0\right\} /(q-\bar{q}) .
$$

It will be useful to define the symbol $a_{0}$ to equal 1 .

The Lax operator of the Toda lattice is the difference operator

$$
L=\Lambda+\sum_{k=1}^{\infty} a_{k} \Lambda^{-k+1} \in \Phi_{-}(\mathcal{A}, q)
$$


its conjugate $\bar{L}$ is given by the formula

$$
\bar{L}=q \Lambda^{-1}+\sum_{k=1}^{\infty} \bar{a}_{k} q^{-[k-1]} \Lambda^{k-1} \in \Phi_{+}(\mathcal{A}, q) .
$$

Introduce elements $p_{k}(n) \in \mathcal{A}$, defined for all $n \geq 0$ and $k \in \mathbb{Z}$ :

$$
L^{n}=\sum_{k=-\infty}^{n} p_{k}(n) \Lambda^{k} .
$$

To define the evolutionary derivation $\delta_{n}$ on the generators $a_{k}$ of $\mathcal{A}$, introduce the difference operator $B_{n}=L_{+}^{n}$, and impose the Lax equation $\delta_{n} L=$ $\varepsilon^{-1}\left[B_{n}, L\right]$. This equation means that

$$
\varepsilon^{-1}\left[B_{n}, L\right]=\sum_{k=1}^{\infty} \delta_{n} a_{k} \Lambda^{-k+1} .
$$

In order for this to be meaningful, it must be shown that the coefficient of $\Lambda^{k}$ in $\left[B_{n}, L\right]$ vanishes for $k>0$. This follows from the identity $\left[L^{n}, L\right]=0$ : we have

$$
\left[B_{n}, L\right]=\left[B_{n}, L\right]-\left[L^{n}, L\right]=-\left[L_{-}^{n}, L\right],
$$

and it is clear that the coefficient of $\Lambda^{k}$ in $\left[L_{-}^{n}, L\right]$ vanishes if $k>0$. We also see that $\delta_{n} a_{k}$ equals the coefficient of $\Lambda^{-k+1}$ in

$$
-\varepsilon^{-1} \sum_{j=0}^{k}\left[p_{j-k}(n) \Lambda^{j-k}, a_{j} \Lambda^{-j+1}\right],
$$

hence that

$$
\begin{aligned}
& \delta_{n} a_{k}-\nabla p_{-k}(n) \\
& =\varepsilon^{-1} \sum_{j=1}^{k-1}\left(\mathrm{E}^{(1-j) / 2} p_{j-k}(n) \mathrm{E}^{(k-j) / 2} a_{j}-\mathrm{E}^{(j-1) / 2} p_{j-k}(n) \mathrm{E}^{(j-k) / 2} a_{j}\right) \\
& =\sum_{j=1}^{k-1}\left(\mathrm{E}^{(1-j) / 2} p_{j-k}(n) \nabla[k-j] a_{j}-\mathrm{E}^{(j-k) / 2} a_{j} \nabla[j-1] p_{j-k}(n)\right) .
\end{aligned}
$$

In particular, $\delta_{n} a_{1}=\nabla p_{-1}(n)$.

To define $\delta_{n}$ on the remaining generators $q$ and $\bar{a}_{k}$ of $\mathcal{A}$, we impose the Lax equation $\delta_{n} \bar{L}=\varepsilon^{-1}\left[B_{n}, \bar{L}\right]$. In particular, we see that

$$
\delta_{n} q=q \nabla p_{0}(n),
$$


and hence that $\delta_{n} q^{[k]}=q^{[k]} \nabla[k] p_{0}(n)$. It also follows that

$$
\delta_{n}\left(q^{-[k-1]} \bar{a}_{k}\right)=q^{-[k-1]}\left(\delta_{n} \bar{a}_{k}-\bar{a}_{k} \nabla[k-1] p_{0}(n)\right)
$$

equals the coefficient of $\Lambda^{k-1}$ in

$$
\varepsilon^{-1} \sum_{j=0}^{k}\left[p_{k-j}(n) \Lambda^{k-j}, q^{-[j-1]} \bar{a}_{j} \Lambda^{j-1}\right],
$$

hence that

$$
\delta_{n} \bar{a}_{k}=\bar{a}_{k} \nabla[k-1] p_{0}(n)+\varepsilon^{-1} q^{[k-1]} \sum_{j=0}^{k} A_{k, j},
$$

where

$$
\begin{aligned}
A_{k, j} & =\mathrm{E}^{(1-j) / 2} p_{k-j}(n) \mathrm{E}^{(k-j) / 2} q^{-[j-1]} \bar{a}_{j}-\mathrm{E}^{(j-1) / 2} p_{k-j}(n) \mathrm{E}^{(j-k) / 2} q^{-[j-1]} \bar{a}_{j} \\
& =\mathrm{E}^{(1-j) / 2}\left(q^{[k-j]} p_{k-j}(n)\right) \mathrm{E}^{(k-j) / 2} \bar{a}_{j}-\mathrm{E}^{(j-1) / 2}\left(q^{[k-j]} p_{k-j}(n)\right) \mathrm{E}^{(j-k) / 2} \bar{a}_{j} .
\end{aligned}
$$

Thus, we see that

$$
\begin{gathered}
\delta_{n} \bar{a}_{k}=\nabla\left(q^{[k]} p_{k}(n)\right)+\sum_{j=1}^{k-1}\left(\mathrm{E}^{(1-j) / 2}\left(q^{[k-j]} p_{k-j}(n)\right) \nabla[k-j] \bar{a}_{j}\right. \\
\left.-\mathrm{E}^{(j-k) / 2} \bar{a}_{j} \nabla[j-1]\left(q^{[k-j]} p_{k-j}(n)\right)\right) .
\end{gathered}
$$

In particular, $\delta_{n} \bar{a}_{1}=\nabla\left(q p_{1}(n)\right)$.

We now recall the proof that the derivations $\delta_{m}$ and $\delta_{n}$ commute. The proof relies on the Zakharov-Shabat equation

$$
\delta_{m} B_{n}-\delta_{n} B_{m}=\varepsilon^{-1}\left[B_{m}, B_{n}\right] .
$$

To prove this equation, observe that

$$
\begin{aligned}
\delta_{m} B_{n} & =\left(\delta_{m} L^{n}\right)_{+}=\varepsilon^{-1}\left[B_{m}, L^{n}\right]_{+}=\varepsilon^{-1}\left[B_{m}, B_{n}+L_{-}^{n}\right]_{+} \\
& =\varepsilon^{-1}\left[B_{m}, B_{n}\right]+\varepsilon^{-1}\left[B_{m}, L_{-}^{n}\right]_{+} .
\end{aligned}
$$

Since $\left[L^{m}, L^{n}\right]=0$, we also see that

$$
\left[B_{m}, L^{n}\right]_{+}=\left[L^{m}-L_{-}^{m}, L^{n}\right]_{+}=-\left[L_{-}^{m}, B_{n}\right]_{+} .
$$

It follows that

$$
\delta_{m} B_{n}-\delta_{n} B_{m}=\varepsilon^{-1}\left(\left[B_{m}, B_{n}\right]+\left[B_{m}, L_{-}^{n}\right]_{+}\right)+\varepsilon^{-1}\left[L_{-}^{n}, B_{m}\right]_{+}=\varepsilon^{-1}\left[B_{m}, B_{n}\right] .
$$


From (10), we easily see that the derivations $\delta_{m}$ and $\delta_{n}$ commute:

$$
\begin{aligned}
{\left[\delta_{m}, \delta_{n}\right] L } & =\varepsilon^{-1} \delta_{m}\left[B_{n}, L\right]-\varepsilon^{-1} \delta_{n}\left[B_{m}, L\right] \\
& =\varepsilon^{-1}\left[\delta_{m} B_{n}, L\right]+\varepsilon^{-2}\left[B_{n},\left[B_{m}, L\right]\right]-\varepsilon^{-1}\left[\delta_{n} B_{m}, L\right]-\varepsilon^{-2}\left[B_{m},\left[B_{n}, L\right]\right] \\
& =\varepsilon^{-1}\left[\delta_{m} B_{n}-\delta_{n} B_{m}, L\right]+\varepsilon^{-2}\left[B_{n},\left[B_{m}, L\right]\right]-\varepsilon^{-2}\left[B_{m},\left[B_{n}, L\right]\right]=0 .
\end{aligned}
$$

The derivation $\bar{\delta}_{n}$ is defined to be the conjugate of $\delta_{n}$, acting on the generators of $\mathcal{A}$ by the formulas

$$
\bar{\delta}_{n} q=\overline{\delta_{n} q}, \quad \bar{\delta}_{n} a_{k}=\overline{\delta_{n} \bar{a}_{k}}, \quad \bar{\delta}_{n} \bar{a}_{k}=\overline{\delta_{n} a_{k}} .
$$

The following proposition establishes the Lax equation for this derivation.

Proposition 2.1. Let $C_{n}=-\bar{L}_{-}^{n}$; then $\bar{\delta}_{n} L=\varepsilon^{-1}\left[C_{n}, L\right]$ and $\bar{\delta}_{n} \bar{L}=$ $\varepsilon^{-1}\left[C_{n}, \bar{L}\right]$.

Proof. We have

$$
\begin{aligned}
\overline{\bar{\delta}_{n} \bar{L}} & =\overline{\bar{\delta}_{n} q} \Lambda^{-1}+\sum_{k=1}^{\infty} \overline{q^{-[k-1]}\left(\bar{\delta}_{n} \bar{a}_{k}-\bar{a}_{k} q^{-[k-1]} \bar{\delta}_{n} q^{[k-1]}\right) \Lambda^{k-1}} \\
& =\sum_{k=1}^{\infty} \overline{\bar{\delta}_{n} \bar{a}_{k}} \Lambda^{-k+1}+\nabla p_{0}(n) \Lambda-\sum_{k=1}^{\infty} a_{k} \nabla[k-1] p_{0}(n) \Lambda^{-k+1} \\
& =\sum_{k=1}^{\infty} \delta_{n} a_{k} \Lambda^{-k+1}-\left[p_{0}(n), L\right]=\varepsilon^{-1}\left[B_{n}, L\right]-\left[p_{0}(n), L\right]=\varepsilon^{-1} \overline{\left[C_{n}, \bar{L}\right]} .
\end{aligned}
$$

A similar proof shows that $\overline{\bar{\delta}_{n} L}=\varepsilon^{-1} \overline{\left[C_{n}, L\right]}$.

It is automatic that the derivations $\bar{\delta}_{m}$ and $\bar{\delta}_{n}$ commute, since their conjugates do. To see that $\delta_{m}$ commutes with $\bar{\delta}_{n}$, we use the Zakharov-Shabat equation

$$
\delta_{m} \bar{B}_{n}-\bar{\delta}_{n} B_{m}=\varepsilon^{-1}\left[B_{m}, \bar{B}_{n}\right] .
$$

This is proved by combining the equations

$$
\delta_{m} \bar{B}_{n}=\left(\delta_{m} \bar{L}^{n}\right)_{-}=\varepsilon^{-1}\left[B_{m}, \bar{L}^{n}\right]_{-}=\varepsilon^{-1}\left[B_{m}, \bar{B}_{n}\right]_{-},
$$

and

$$
\bar{\delta}_{m} B_{n}=\left(\bar{\delta}_{n} L^{m}\right)_{+}=\varepsilon^{-1}\left[\bar{B}_{n}, L^{m}\right]_{+}=\varepsilon^{-1}\left[\bar{B}_{n}, B_{m}\right]_{+} .
$$


It follows from (11) that $\delta_{m}$ and $\bar{\delta}_{n}$ commute:

$$
\begin{aligned}
{\left[\delta_{m}, \bar{\delta}_{n}\right] L } & =\varepsilon^{-1} \delta_{m}\left[\bar{B}_{n}, L\right]-\varepsilon^{-1} \bar{\delta}_{n}\left[B_{m}, L\right] \\
& =\varepsilon^{-1}\left[\delta_{m} \bar{B}_{n}, L\right]+\varepsilon^{-2}\left[\bar{B}_{n},\left[B_{m}, L\right]\right]-\varepsilon^{-1}\left[\bar{\delta}_{n} B_{m}, L\right]-\varepsilon^{-2}\left[B_{m},\left[\bar{B}_{n}, L\right]\right] \\
& =\varepsilon^{-1}\left[\delta_{m} \bar{B}_{n}-\bar{\delta}_{n} B_{m}, L\right]+\varepsilon^{-2}\left[\bar{B}_{n},\left[B_{m}, L\right]\right]-\varepsilon^{-2}\left[B_{m},\left[\bar{B}_{n}, L\right]\right]=0 .
\end{aligned}
$$

Denote by $\alpha: \mathcal{A} \rightarrow \mathbb{Q}_{\varepsilon}$ the homomorphism which sends the generators $\left\{q, a_{k}, \bar{a}_{k}\right\}$ of $\mathcal{A}$ to 0 . By formulas (7), (8) and (9), we see that $\delta_{n} q, \delta_{n} a_{k}$ and $\delta_{n} \bar{a}_{k}$ all lie in the ideal $(\partial \mathcal{A})$ of $\mathcal{A}$, and hence

$$
\alpha \cdot \delta_{n}=0 .
$$

\section{§3. The Equivariant Toda Lattice}

Informally, a reduction of the Toda lattice is an invariant submanifold of the configuration space fixed by the involution; we formalize this as follows.

Definition 3.1. A reduction of the Toda lattice is a differential ideal $\mathcal{I} \subset \mathcal{A}$ invariant under conjugation and preserved by the action of the derivations $\delta_{n}$ and $\bar{\delta}_{n}$.

The simplest example of such a reduction is the Toda chain, defined by the constraint $L=\bar{L}$; the associated differential ideal

$$
\mathcal{I}=\left(a_{1}-\bar{a}_{1}, a_{2}-q, a_{k} \mid k>2\right)
$$

is generated by the coefficients of $L-\bar{L}$. To see that $\mathcal{I}$ is closed under the action of the derivations $\delta_{n}$, it suffices to observe that the operator $L-\bar{L}$ satisfies the Lax equation $\delta_{n}(L-\bar{L})=\varepsilon^{-1}\left[B_{n}, L-\bar{L}\right]$, and that the coefficients of $\varepsilon^{-1}\left[B_{n}, L-\bar{L}\right]$ are contained in the differential ideal generated by the coefficients of $L-\bar{L}$.

The constraint $L=\bar{L}$ is equivalent to the relation $\delta_{n}=\bar{\delta}_{n}$ among the Toda flows, for all $n$; in particular, the Toda equation (2) becomes in this limit the equation $\delta_{1}^{2} \log q=\nabla^{2} q$.

In this paper, we study a reduction of the Toda lattice which is a deformation of the Toda chain. Let $\mathcal{A}[\nu]$ be the extension of the differential algebra $\mathcal{A}$ by a variable $\nu$, such that $\partial \nu=0$ and $\bar{\nu}=-\nu$, and consider families of reductions of the Toda lattice parametrized by $\mathcal{A}[\nu]$; that is, we consider differential ideals in $\mathcal{A}[\nu]$ satisfying the conditions of Definition 3.1. 
Definition 3.2. The equivariant Toda lattice is the reduction of the Toda lattice defined over $\mathcal{A}[\nu]$ by the constraints

$$
\left(\delta_{1}-\bar{\delta}_{1}\right) L=\nu \partial L, \quad\left(\delta_{1}-\bar{\delta}_{1}\right) \bar{L}=\nu \partial \bar{L} .
$$

Let $\mathcal{I}_{\nu}$ be the differential ideal determining this reduction.

Let $K$ be the difference operator

$$
K=B_{1}-C_{1}=\Lambda+a_{1}+q \Lambda^{-1} .
$$

Substituting the Lax equations into the constraints (13), we obtain an equivalent formulation of the equivariant Toda lattice: it is characterized by the pair of equations

$$
\varepsilon^{-1}[K, L]=\nu \partial L, \quad \varepsilon^{-1}[K, \bar{L}]=\nu \partial \bar{L} .
$$

In other words, the differential ideal $\mathcal{I}_{\nu}$ defining the equivariant Toda lattice is generated by the coefficients of these equations.

The following theorem collects the main properties of the differential ideal $\mathcal{I}_{\nu}$. Let $\mathrm{P}: \mathcal{A} \rightarrow \mathcal{A}$ be the infinite-order differential operator

$$
\begin{aligned}
\mathrm{P} & =\frac{\partial}{\nabla}=\sum_{g=0}^{\infty} \frac{\varepsilon^{2 g}\left(2^{1-2 g}-1\right) B_{2 g}}{(2 g) !} \partial^{2 g} \\
& =1-\frac{1}{24} \varepsilon^{2} \partial^{2}+O\left(\varepsilon^{4}\right) .
\end{aligned}
$$

Theorem 3.1. The differential ideal $\mathcal{I}_{\nu}$ defining the equivariant Toda lattice equals $\left(\eta, \partial \zeta_{k} \mid k>0\right)$, where

$$
\begin{aligned}
\eta & =q \nabla\left(a_{1}-\bar{a}_{1}\right)-\nu \partial q, \\
\zeta_{k} & =p_{-1}(k)-q p_{1}(k)-\nu \mathrm{P} p_{0}(k) .
\end{aligned}
$$

The differential algebra $\mathcal{A}[\nu] / \mathcal{I}_{\nu}$ is isomorphic to

$$
\widetilde{\mathcal{A}}=\mathbb{Q}_{\varepsilon, \nu}\left\{q, v, z_{k} \mid k>0\right\} /\left(q-\bar{q}, y, \partial z_{k} \mid k>0\right),
$$

where $v$ and $z_{k}$ are identified with the images of $a_{1}$ and $\zeta_{k}$ in $\mathcal{A}[\nu] / \mathcal{I}_{\nu}$, and $y=q \nabla(v-\bar{v})-\nu \partial q$.

The Toda flows $\delta_{n}$ and $\bar{\delta}_{n}$ map $\zeta_{k} \in \mathcal{A}[\nu]$ to $\mathcal{I}_{\nu}$; in particular, the variables $z_{k} \in \widetilde{\mathcal{A}}$ are constants of motion for the flows of the Toda lattice. 
Proof. Let $\widetilde{\mathcal{I}}_{\nu}$ be the differential ideal $\left(\eta, \partial \zeta_{k} \mid k>0\right)$. Define elements $f_{k}(n), g_{k}(n) \in \mathcal{A}$ by the formulas

$$
\varepsilon^{-1}\left[K, L^{n}\right]-\nu \partial L^{n}=\sum_{k=-\infty}^{\infty} f_{k}(n) \Lambda^{-k}, \varepsilon^{-1}\left[K, \bar{L}^{n}\right]-\nu \partial \bar{L}^{n}=\sum_{k=-\infty}^{\infty} g_{k}(n) \Lambda^{k}
$$

The differential ideal $\mathcal{I}_{\nu}$ is generated by the coefficients $f_{k}=f_{k}(1)$ and $g_{k}=$ $g_{k}(1)$. The formulas

$$
\begin{aligned}
& \varepsilon^{-1}\left[K, L^{n}\right]-\nu \partial L^{n}=\sum_{i=1}^{n} L^{i-1}\left(\varepsilon^{-1}[K, L]-\nu \partial L\right) L^{n-i}, \\
& \varepsilon^{-1}\left[K, \bar{L}^{n}\right]-\nu \partial \bar{L}^{n}=\sum_{i=1}^{n} \bar{L}^{i-1}\left(\varepsilon^{-1}[K, \bar{L}]-\nu \partial \bar{L}\right) \bar{L}^{n-i},
\end{aligned}
$$

show that the coefficients $f_{k}(n)$ and $g_{k}(n)$ lie in $\mathcal{I}_{\nu}$; hence $\partial \zeta_{n}=\operatorname{P} f_{0}(n)$ and $\partial \bar{\zeta}_{n}=\mathrm{P} g_{0}(n)$ do as well, showing that $\widetilde{\mathcal{I}}_{\nu} \subset \mathcal{I}_{\nu}$. We wish to prove the equality of these two differential ideals.

To do this, we show, by induction on $k$, that the coefficients $f_{k}$ lie in $\widetilde{\mathcal{I}}_{\nu}$. We have $f_{k}=0$ for $k<0$. If $f_{j} \in \widetilde{\mathcal{I}}_{\nu}$ for $j<k$, we see that

$$
\begin{aligned}
\nabla z_{k+1} & =\operatorname{res}\left(\varepsilon^{-1}\left[K, L^{k+1}\right]-\nu \partial L^{k+1}\right)=\sum_{i=0}^{k} \operatorname{res}\left(L^{i}\left(\varepsilon^{-1}[K, L]-\nu \partial L\right) L^{k-i}\right) \\
& \equiv[k+1] f_{k} \quad\left(\bmod \widetilde{\mathcal{I}}_{\nu}\right),
\end{aligned}
$$

hence $f_{k} \in \widetilde{\mathcal{I}}_{\nu}$. A similar induction shows that $g_{k} \in \widetilde{\mathcal{I}}_{\nu}$; this induction starts with the fact that $g_{-1}=\eta$ lies in $\tilde{\mathcal{I}}_{\nu}$.

Since $\zeta_{k}-[k] a_{k+1} \in\left(q, a_{1}, \ldots, a_{k}\right)$, we see that the differential algebra $\mathcal{A} / \mathcal{I}_{\nu}$ is isomorphic to

$$
\widetilde{\mathcal{A}}=\mathbb{Q}_{\varepsilon, \nu}\left\{q, v, z_{k} \mid k>0\right\} /\left(q-\bar{q}, y, \partial z_{k} \mid k>0\right) .
$$

It remains to prove that $\delta_{n} \zeta_{k}$ and $\bar{\delta}_{n} \zeta_{k}$ lie in $\mathcal{I}_{\nu}$. By the Zakharov-Shabat equations (10) and (11), we see that

$$
\begin{aligned}
\delta_{n} K & =\delta_{n}\left(B_{1}-C_{1}\right)=\left(\delta_{1}-\bar{\delta}_{1}\right) B_{n}+\varepsilon^{-1}\left[B_{n}, B_{1}-C_{1}\right] \\
& =\nu \partial B_{n}+\varepsilon^{-1}\left[B_{n}, K\right] .
\end{aligned}
$$


It follows that

$$
\begin{aligned}
\nabla \delta_{n} \zeta_{k} & =\delta_{n} f_{0}(k)=\delta_{n} \operatorname{res}\left(\varepsilon^{-1}\left[K, L^{k}\right]-\nu \partial L^{k}\right) \\
& =\operatorname{res}\left(\varepsilon^{-1}\left[\delta_{n} K, L^{k}\right]+\varepsilon^{-1}\left[K, \delta_{n} L^{k}\right]-\nu \partial \delta_{n} L^{k}\right) \\
& =\varepsilon^{-1} \operatorname{res}\left(\left[\nu \partial B_{n}+\varepsilon^{-1}\left[B_{n}, K\right], L^{k}\right]+\varepsilon^{-1}\left[K,\left[B_{n}, L^{k}\right]\right]-\nu \partial\left[B_{n}, L^{k}\right]\right) \\
& =\varepsilon^{-1} \operatorname{res}\left(\left[B_{n}, \varepsilon^{-1}\left[K, L^{k}\right]-\nu \partial L^{k}\right]\right) \\
& =\nabla \sum_{j=1}^{n}[j]\left(p_{j}(n) f_{j}(k)\right) .
\end{aligned}
$$

The extension of $\alpha$ to a homomorphism from $\mathcal{A}[\nu]$ to $\mathbb{Q}_{\varepsilon, \nu}$ continues to satisfy (12). It follows that $\alpha\left(\delta_{n} \zeta_{k}\right)=0$, hence we obtain an explicit equation for $\delta_{n} \zeta_{k}$ :

$$
\delta_{n} \zeta_{k}=\sum_{j=1}^{n}[j]\left(p_{j}(n) f_{j}(k)\right) \in \mathcal{I}_{\nu} .
$$

The proof that $\bar{\delta}_{n} \zeta_{k} \in \mathcal{I}_{\nu}$ follows along the same lines.

Let us illustrate this theorem by calculating the coefficients $a_{2}$ and $a_{3}$ of the Lax operator $L$ as elements of $\widetilde{\mathcal{A}}$. Applying res $: \Phi_{-}(\mathcal{A}, q) \rightarrow \mathcal{A}$ to the equation $\varepsilon^{-1}[K, L]=\nu \partial L$, we see that

$$
a_{2}=q+\nu \mathrm{P} v+z_{1} .
$$

Taking the coefficient of $\Lambda^{-1}$ in the equation $\varepsilon^{-1}[K, L]=\nu \partial L$, we see that

$$
\nabla a_{3}+\left(a_{2}-q\right) \nabla v=\nu \partial a_{2} .
$$

Lemma 3.1. $\quad \nabla f \mathrm{P} g=\frac{1}{2} \nabla(f[2] \mathrm{P} g)-\frac{1}{2}[2](f \partial g)$

Proof. We have

$$
\nabla f \mathrm{P} g=\varepsilon^{-1} \mathrm{E}^{1 / 2}\left(f \mathrm{E}^{-1 / 2} \mathrm{P} g\right)-\varepsilon^{-1} \mathrm{E}^{-1 / 2}\left(f \mathrm{E}^{1 / 2} \mathrm{P} g\right) .
$$

The result follows, since $\mathrm{E}^{ \pm 1 / 2} \mathrm{P}=\frac{1}{2}[2] \mathrm{P} \pm \frac{1}{2} \varepsilon \partial$.

By this lemma,

$$
\left(a_{2}-q\right) \nabla v=\nu \nabla v \mathrm{P} v+z_{1} \nabla v=\nu \nabla\left(\frac{1}{2} v[2] \mathrm{P} v-\frac{1}{4} \mathrm{P}[2] v^{2}\right)+z_{1} \nabla v .
$$

It follows that

$$
a_{3}=\nu\left(\mathrm{P}\left(\frac{1}{4}[2] v^{2}+q\right)-\frac{1}{2} v[2] \mathrm{P} v\right)+\nu^{2} \mathrm{P} v-z_{1} v+\frac{1}{2} z_{2} .
$$


This method of calculating the coefficients $a_{k}$ becomes cumbersome for larger values of $k$ : instead, it is better to use the recursion in $\widetilde{\mathcal{A}}$

$$
p_{-1}(n)=q p_{1}(n)+\nu \mathrm{P} p_{0}(n)+z_{n}
$$

which is a consequence of Theorem 3.1.

Let $\Psi$ be the algebra of difference operators

$$
\Psi=\left\{A \in \Phi_{-}(\widetilde{\mathcal{A}}, q) \mid \varepsilon^{-1}[K, A]=\nu \partial A\right\} .
$$

Let $\mathbb{L} \in \Psi$ be the Lax operator defined by the recursion

$$
p_{-1}(n)=q p_{1}(n)+\nu \mathrm{P} p_{0}(n) .
$$

This Lax operator plays a special role in the theory: the following lemma shows that the algebra $\Psi$ may be identified with the commutative algebra $\widetilde{\mathcal{A}}_{0}((\mathbb{L}))$, where

$$
\widetilde{\mathcal{A}}_{0}=\mathbb{Q}_{\varepsilon, \nu}\left[z_{k}, \bar{z}_{k} \mid k>0\right]
$$

is the kernel of the derivation $\partial: \widetilde{\mathcal{A}} \rightarrow \widetilde{\mathcal{A}}$.

Lemma 3.2. The homomorphism $\tilde{\alpha}: \widetilde{\mathcal{A}} \rightarrow \widetilde{\mathcal{A}_{0}}$ which sends the generators $\partial^{n} q, \partial^{n} v$ and $\partial^{n} \bar{v}$ of $\widetilde{\mathcal{A}}$ to 0 induces an isomorphism between $\Psi$ and $\widetilde{\mathcal{A}}_{0}\left(\left(\Lambda^{-1}\right)\right)$.

Proof. Since $\tilde{\alpha}(\mathbb{L})=\Lambda$, the map $\tilde{\alpha}: \Psi \rightarrow \widetilde{\mathcal{A}}_{0}\left(\left(\Lambda^{-1}\right)\right)$ is surjective. Suppose that $A \in \Psi$ lies in the kernel of $\tilde{\alpha}$, and let $k$ be the smallest integer such that the coefficient $x \in \widetilde{\mathcal{A}}$ of $\Lambda^{-k}$ in $A$ is nonzero. We have

$$
\varepsilon^{-1}[K, A]-\nu \partial A=\nabla x \Lambda^{1-k}+O\left(\Lambda^{-k}\right),
$$

hence $x \in \widetilde{\mathcal{A}}_{0}$. In this way, we see that $\tilde{\alpha}: \Psi \rightarrow \widetilde{\mathcal{A}}_{0}\left(\left(\Lambda^{-1}\right)\right)$ is injective.

Theorem 3.2. The evolutionary derivation $e=\partial_{v}+\partial_{\bar{v}}$ of $\widetilde{\mathcal{A}}$ preserves $\Psi$, and $e(L)$ satisfies the formula

$$
\left(L-\nu+\sum_{k=1}^{\infty} z_{k} L^{-k}\right) e(L)=L .
$$

Proof. If $A \in \Psi$, we have

$$
\varepsilon^{-1}[K, e(A)]-\nu \partial e(A)=e\left(\varepsilon^{-1}[K, A]-\nu \partial A\right)-[e(K), A]=0,
$$

since $e(K)=1$. This shows that $e$ preserves $\Psi$. 
For $n>0$, we have by $(19)$ that $\tilde{\alpha}\left(e\left(p_{-1}(n)\right)\right)=\nu \tilde{\alpha}\left(e\left(p_{0}(n)\right)\right)$, or equivalently,

$$
\oint(\mathbb{L}-\nu) e\left(\mathbb{L}^{n}\right) \frac{d \mathbb{L}}{\mathbb{L}}=0 .
$$

Since $\Psi$ is a commutative algebra, $e(\mathbb{L})$ commutes with $\mathbb{L}$, hence $e\left(\mathbb{L}^{n}\right)=$ $n \mathbb{L}^{n-1} e(\mathbb{L})$, and

$$
\oint(\mathbb{L}-\nu) \mathbb{L}^{n-2} e(\mathbb{L}) d \mathbb{L}=0, \quad n>0 .
$$

This shows that the coefficient of $\mathbb{L}^{-k}$ in $(\mathbb{L}-\nu) e(\mathbb{L})$ vanishes, hence $(\mathbb{L}-\nu) e(\mathbb{L})=\mathbb{L}$.

Since $L / e(L)$ lies in $\Psi$, there is an expansion

$$
\frac{L}{e(L)}=L-\nu+\frac{1}{2 \pi i} \sum_{n=0}^{\infty} L^{-n-1} \oint L^{n} \frac{d L}{e(L)} .
$$

(The constant term is determined by the fact that $e(L)=1+\nu \Lambda^{-1}+O\left(\Lambda^{-2}\right)$.) We have

$$
\frac{d L}{e(L)}=\frac{d \mathbb{L}}{e(\mathbb{L})}=(\mathbb{L}-\nu) \frac{d \mathbb{L}}{\mathbb{L}}
$$

hence

$$
\frac{1}{2 \pi i} \oint L^{n} \frac{d L}{e(L)}=\frac{1}{2 \pi i} \oint L^{n}(\mathbb{L}-\nu) \frac{d \mathbb{L}}{\mathbb{L}} .
$$

It follows from the recursion (18) that

$$
z_{n}=\tilde{\alpha}\left(p_{-1}(n)\right)-\nu \tilde{\alpha}\left(p_{0}(n)\right)=\frac{1}{2 \pi i} \oint L^{n}(\mathbb{L}-\nu) \frac{d \mathbb{L}}{\mathbb{L}},
$$

and the theorem follows.

\section{§4. The Dispersionless Limit of the Equivariant Toda Lattice}

In this section, we consider the dispersionless limit of the equivariant Toda lattice, in which $\varepsilon \rightarrow 0$; we only consider the case in which the constants of motion $z_{k}$ are set to 0 . If $A \in \Phi_{ \pm}(\widetilde{\mathcal{A}}, q)$, we write

$$
A_{0}=\lim _{\varepsilon \rightarrow 0} A \in \mathbb{Q}[\nu, q, v]\left(\left(\Lambda^{-1}\right)\right) .
$$


In the dispersionless limit, the algebra $\Phi_{-}(\widetilde{\mathcal{A}}, q)$ degenerates to the commutative algebra $\widetilde{\mathcal{A}}\left(\left(\Lambda^{-1}\right)\right)$, and the leading order in the commutator is the Poisson bracket

$$
\left\{A_{0}, B_{0}\right\}=\lim _{\varepsilon \rightarrow 0} \varepsilon^{-1}[A, B]=\left(\Lambda \partial_{\Lambda} A_{0}\right) \partial B_{0}-\partial A_{0}\left(\Lambda \partial_{\Lambda} B_{0}\right) .
$$

It is not hard to write down explicit formulas for the Lax operator $\mathbb{L}$ of the equivariant Toda lattice and its conjugate $\overline{\mathbb{L}}$ in the dispersionless limit.

Define the (unsigned) Stirling numbers (of the first kind) $\left[\begin{array}{l}n \\ k\end{array}\right]$ by the generating function

$$
\sum_{k=0}^{n}\left[\begin{array}{l}
n \\
k
\end{array}\right] \nu^{k}=\prod_{j=0}^{n-1}(\nu+j)
$$

We have the recursion

$$
\left[\begin{array}{l}
n \\
k
\end{array}\right]=(n-1)\left[\begin{array}{c}
n-1 \\
k
\end{array}\right]+\left[\begin{array}{l}
n-1 \\
k-1
\end{array}\right] .
$$

Theorem 4.1. We have

$$
\begin{aligned}
& \mathbb{L}_{0}=K_{0}+\nu \sum_{n=0}^{\infty}\left(\frac{\nu}{\Lambda}\right)^{n} \sum_{k=0}^{n}(-1)^{n-k}\left[\begin{array}{l}
n \\
k
\end{array}\right] \frac{\log \left(K_{0} / \Lambda\right)^{n-k+1}}{(n-k+1) !}\left(\frac{K_{0}}{\Lambda}\right)^{-n}, \text { and } \\
& \overline{\mathbb{L}}_{0}=K_{0}-\nu \sum_{n=0}^{\infty}\left(\frac{-\nu \Lambda}{q}\right)^{n} \sum_{k=0}^{n}(-1)^{n-k}\left[\begin{array}{l}
n \\
k
\end{array}\right] \frac{\log \left(\Lambda K_{0}\right)^{n-k+1}}{(n-k+1) !}\left(\frac{\Lambda K_{0}}{q}\right)^{-n}
\end{aligned}
$$

Proof. Denote by $L_{0}$ the expression which we wish to prove equals $\mathbb{L}_{0}$. It is clear that $\tilde{\alpha}(L)=\Lambda$, hence it suffices to prove the equation

$$
\left\{K_{0}, L_{0}\right\}=\nu \partial K_{0}
$$

which is the dispersionless limit of the equation $\varepsilon^{-1}[K, \mathbb{L}]=\nu \partial \mathbb{L}$.

Since $\left\{K_{0}, \log \left(K_{0} / \Lambda\right)\right\}=\partial K_{0}$, we have

$$
\left\{K_{0}, L_{0}\right\}=\nu \partial K_{0} \sum_{n=0}^{\infty}\left(\frac{\nu}{\Lambda}\right)^{n} \sum_{k=0}^{n}(-1)^{n-k}\left[\begin{array}{l}
n \\
k
\end{array}\right] \frac{\log \left(K_{0} / \Lambda\right)^{n-k}}{(n-k) !}\left(\frac{K_{0}}{\Lambda}\right)^{-n}
$$

Since $\partial \log \left(K_{0} / \Lambda\right)=K_{0}^{-1} \partial K_{0}$, we have

$$
\partial L_{0}=\partial K_{0}\left(1+\sum_{n=0}^{\infty}\left(\frac{\nu}{\Lambda}\right)^{n} \sum_{k=0}^{n}(-1)^{n-k} A_{n, k}\left[\begin{array}{l}
n \\
k
\end{array}\right]\left(\frac{K_{0}}{\Lambda}\right)^{-n-1}\right),
$$


where

$$
A_{n, k}=\frac{\log \left(K_{0} / \Lambda\right)^{n-k}}{(n-k) !}-\frac{n \log \left(K_{0} / \Lambda\right)^{n-k+1}}{(n-k+1) !} .
$$

The equation $\left\{K_{0}, L_{0}\right\}=\nu \partial L_{0}$ follows from $(20)$.

Define $e^{\circ j}\left(L_{0}\right)$ by induction: $e^{\circ 0}\left(L_{0}\right)=L_{0}$ and $e^{\circ(j+1)}\left(L_{0}\right)=e\left(e^{\circ j}\left(L_{0}\right)\right)$. Then

$$
\begin{aligned}
e^{\circ j}\left(L_{0}\right)= & \delta_{j, 0} K_{0} \\
& +\nu^{1-j} \sum_{n=0}^{\infty}\left(\frac{\nu}{\Lambda}\right)^{n} \sum_{k=0}^{n-j+1}(-1)^{n-k-j}\left[\begin{array}{l}
n \\
k
\end{array}\right] \frac{\log \left(K_{0} / \Lambda\right)^{n-k-j+1}}{(n-k-j+1) !}\left(\frac{K_{0}}{\Lambda}\right)^{-n} .
\end{aligned}
$$

This formula is proved by induction on $j$, using the formulas $e\left(K_{0}\right)=1$ and $e\left(\log \left(K_{0} / \Lambda\right)\right)=K_{0}^{-1}$.

There is an embedding of the differential algebra $\widetilde{\mathcal{A}}$ in the differential algebra

$$
\widetilde{\mathcal{A}}\{u\} /(\partial q-q \partial u) \cong \mathbb{Q}_{\varepsilon, \nu}[q]\{u, v\}
$$

given by mapping $\bar{v}$ to $v-\nu \mathrm{P} u$. In the dispersionless limit, this embedding maps $\bar{v}$ to $v-\nu u$. We will prove the formula for $\overline{\mathbb{L}}_{0}$ by working with Laurent series in this larger algebra.

The Laurent series $\overline{\mathbb{L}}_{0}$ is obtained from $\mathbb{L}_{0}$ by replacing $v$ by $v-\nu u, \Lambda$ by $q / \Lambda$, and $\nu$ by $-\nu$. Let $\widetilde{\mathbb{L}}_{0}$ be the result of substituting $v-\nu u$ for $v$ in $\mathbb{L}_{0}$; it is given by the formula

$$
\begin{aligned}
\widetilde{\mathbb{L}}_{0} & =\sum_{j=0}^{\infty} \frac{(-\nu u)^{j}}{j !} e^{\circ j}(L) \\
& =K_{0}+\nu \sum_{n=0}^{\infty}\left(\frac{\nu}{\Lambda}\right)^{n} \sum_{j=0}^{n+1} \sum_{k=0}^{n-j+1}(-1)^{n-k}\left[\begin{array}{l}
n \\
k
\end{array}\right] \frac{u^{j} \log \left(K_{0} / \Lambda\right)^{n-k-j+1}}{j !(n-k-j+1) !}\left(\frac{K_{0}}{\Lambda}\right)^{-n} \\
& =K_{0}+\nu \sum_{n=0}^{\infty}\left(\frac{\nu}{\Lambda}\right)^{n} \sum_{k=0}^{n+1}(-1)^{n-k}\left[\begin{array}{l}
n \\
k
\end{array}\right] \frac{\log \left(q K_{0} / \Lambda\right)^{n-k+1}}{(n-k+1) !}\left(\frac{K_{0}}{\Lambda}\right)^{-n} .
\end{aligned}
$$

We obtain $\overline{\mathbb{L}}_{0}$ on substituting $-\nu$ for $\nu$ and $\Lambda$ for $q / \Lambda$.

We may now prove the formulas (5a) and (5b) relating the dispersionless limit of the equivariant Toda lattice to the equivariant genus 0 Gromov-Witten potential of $\mathbb{C P}^{1}$. 
In the genus 0 limit, the functions $q=\exp (u)$ and $v$ on the large phase space become $\exp \left(\partial^{2} \mathcal{F}_{0}\right)$ and $\partial \partial_{0} \mathcal{F}_{0}$, and $\bar{v}$ becomes $v-\nu u$. The proof of Theorem 4.2 of [5] extends to the equivariant case, and shows that

$$
\partial^{n} u=s_{n}+O\left(|s|^{2}+|t|^{2}\right), \quad \partial^{n} v=\delta_{1, n}+t_{n}+O\left(|s|^{2}+|t|^{2}\right) .
$$

Hence, we may identify the large phase space with the space of formal jets in an affine space with coordinates $u$ and $v$.

The following lemma shows that the vector field $e$ lifts to the puncture vector field on the large phase space.

Lemma 4.1. The puncture vector field

$$
e=\partial-\sum_{n=0}^{\infty}\left(s_{n+1} \frac{\partial}{\partial s_{n}}+t_{n+1} \frac{\partial}{\partial t_{n}}\right)
$$

on the large phase space acts on elements of $\mathbb{Q}[\nu, q, v]$ by the derivation $\partial_{v}$.

Proof. Observe that the puncture vector field $e$ commutes with $\partial 1$; this reflects the fact that $\mathbb{C P}^{1}$ is one-dimensional. The puncture (or string) equation says that

$$
e\left(\mathcal{F}_{0}\right)=s_{0} t_{0}+\frac{1}{2} \nu t_{0}^{2}
$$

Applying the differential operators $\partial_{0} \partial$ and $\partial^{2}$ to this equation, we see that $e(v)=1$ and $e(u)=0$.

Define generating functions $\pi_{k}(z) \in \mathbb{Q}[\nu, q, v]((z))$ by the formula

$$
\mathbb{L}_{0}(z)=\sum_{n=-\infty}^{\infty} \frac{z^{n}}{[n] !} \mathbb{L}_{0}^{n}=\sum_{k=-\infty}^{\infty} \pi_{k}(z) \Lambda^{k}
$$

where $[n]$ ! is the rational function

$$
[n] !=\frac{\Gamma(\nu z+n+1)}{\Gamma(\nu z+1)}=(1+z \nu)(2+z \nu) \ldots(n+z \nu) .
$$

It follows from the recursion (19) for the coefficients of $\mathbb{L}$ that

$$
\pi_{-1}(z)=q \pi_{1}(z)+\nu \pi_{0}(z) .
$$

Lemma 4.2. $\quad e\left(\pi_{k}(z)\right)=z \pi_{k}(z)$ and $\partial_{q} \pi_{k}(z)=z \pi_{k+1}(z)$. 
Proof. We have

$$
\begin{aligned}
\left(\mathbb{L}_{0}-\nu\right) e\left(\mathbb{L}_{0}(z)\right) & =\sum_{n=-\infty}^{\infty} \frac{n z^{n}}{[n] !} \mathbb{L}_{0}^{n-1}\left(\mathbb{L}_{0}-\nu\right) e\left(\mathbb{L}_{0}\right)=\sum_{n=-\infty}^{\infty} \frac{n z^{n}}{[n] !} \mathbb{L}_{0}^{n} \\
& =\sum_{n=-\infty}^{\infty} \frac{(\nu z+n) z^{n}}{[n] !} \mathbb{L}_{0}^{n}-\sum_{n=-\infty}^{\infty} \frac{\nu z^{n+1}}{[n] !} \mathbb{L}_{0}^{n}=z\left(\mathbb{L}_{0}-\nu\right) \mathbb{L}_{0}(z) .
\end{aligned}
$$

The equation for $e\left(\pi_{k}(z)\right)$ follows on taking the coefficient of $\Lambda^{k}$. Since $\Lambda \partial_{q} K_{0}=$ $e\left(K_{0}\right)$, it follows from Theorem 4.1 that $\Lambda \partial_{q} \mathbb{L}_{0}(z)=e\left(\mathbb{L}_{0}(z)\right)$. Taking the coefficient of $\Lambda^{k+1}$, we obtain the formula for $\partial_{q} \pi_{k}(z)$.

Theorem 4.2. The dispersionless limits of (5a) and (5b) hold.

Proof. We will concentrate on the proof of (5a). The proof of the dispersionless limit of $(5 \mathrm{~b})$ is the same, up to conjugation.

Let $\partial(z)$ be the generating function for vector fields

$$
\partial(z)=\sum_{k=0}^{\infty} z^{k} \partial_{k}
$$

We must prove that

$$
\partial(z) v=\sum_{n=1}^{\infty} \frac{z^{n-1} \partial p_{0}(n)}{[n] !}, \quad \partial(z) u=\sum_{n=1}^{\infty} \frac{z^{n-1} \partial p_{-1}(n)}{[n] !} .
$$

In terms of the generating functions

$$
x=1+z \partial(z) \partial \mathcal{F}_{0}-g_{0}(z), \quad y=\nu+z \partial(z) \partial_{0} \mathcal{F}_{0}-g_{-1}(z),
$$

we wish to prove that $\partial x(z)=\partial y(z)=0$. We will actually prove the stronger result, that $x(z)=y(z)=0$ : in other words, that

$$
\sum_{k=0}^{\infty} z^{k} \partial_{k} \partial \mathcal{F}_{0}=\sum_{n=1}^{\infty} \frac{z^{n-1} p_{0}(n)}{[n] !}, \quad \sum_{k=0}^{\infty} z^{k} \partial_{k} \partial_{0} \mathcal{F}_{0}=\sum_{n=1}^{\infty} \frac{z^{n-1} p_{-1}(n)}{[n] !}
$$

A theorem of Dijkgraaf and Witten [2] establishes that the Toda equation (2) holds in the dispersionless limit:

$$
\partial_{0}^{2} \mathcal{F}_{0}=q+\nu v .
$$


Combining the topological recursion relations for equivariant Gromov-Witten invariants in genus 0 with Lemma 4.2, we see that

$$
\begin{aligned}
& \partial x(z)=z(x(z) \partial(v-\nu u)+y(z) \partial u) \\
& \partial y(z)=z(x(z) \partial q+y(z) \partial v) .
\end{aligned}
$$

On the other hand, the string equation shows that $e(x(z))=z x(z)$ and $e(y(z))=z y(z)$.

Now apply the following principle (Proposition 4.1 of [5]):

A function $f$ on the large phase space such that $\partial f$ and $e(f)$ lie in $\mathbb{Q}[\nu]$ itself lies in $\mathbb{Q}[\nu]$.

Arguing by induction, we see that the coefficients of $z^{k}$ in $x(z)$ and $y(z)$ lie in $\mathbb{Q}[\nu]$; in other words, $x(z), y(z) \in \mathbb{Q} \llbracket \nu, z \rrbracket$. (In particular, we see that $\partial_{k} \partial \mathcal{F}_{0}$ and $\partial_{k} \partial_{0} \mathcal{F}_{0}$ lie in $\mathbb{Q}[\nu, q, v]$ for all $k \geq 0$.)

The proof is finished by observing that, by the divisor equation for GromovWitten invariants, the $\operatorname{limits}_{\lim _{q \rightarrow 0}} x(z)$ and $\lim _{q \rightarrow 0} y(z)$ are integrals over the degree 0 moduli space $\overline{\mathcal{M}}_{0,2}\left(\mathbb{C P}^{1}, 0\right)$; however, this moduli space is empty, hence $x(z)=y(z)=0$.

As mentioned in the introduction, the analogue of Theorem 4.2 is now known to hold in all genera (Okounkov and Pandharipande [9]).

\section{$\S 5 . \quad$ The Dressing Operator of the Toda Lattice}

Let $W$ be the universal dressing operator of the Toda lattice

$$
W=1+\sum_{k=1}^{\infty} w_{k} \Lambda^{-k} \in \Phi_{-}(\mathcal{B}, q),
$$

where $\mathcal{B}$ is the free differential algebra $\mathbb{Q}_{\varepsilon}\left\{q, w_{k} \mid k>0\right\} /(q-\bar{q})$. The coefficients $w_{k}^{*} \in \mathcal{B}$ of

$$
W^{-1}=1+\sum_{k=1}^{\infty} w_{k}^{*} \Lambda^{-k}
$$

are characterized by the recursion obtained by extracting the coefficient of $\Lambda^{-k}$ in the equation $W W^{-1}=1$ :

$$
w_{k}^{*}=-w_{k}-\sum_{j=1}^{k-1}\left(\mathrm{E}^{(k-j) / 2} w_{j}\right)\left(\mathrm{E}^{-j / 2} w_{k-j}^{*}\right) .
$$


Lemma 5.1. $\quad$ There is an embedding $\mathcal{A} \hookrightarrow \mathcal{B}$, characterized by the dressing equation $L=W \Lambda W^{-1}$, or equivalently, $L W=W \Lambda$.

Proof. For all $k>0, a_{k}+\varepsilon \nabla w_{k}$ lies in the differential ideal $\left(w_{1}, \ldots, w_{k-1}\right)$.

The conjugate Lax operator $\bar{L}$ is given by the dressing equation

$$
\bar{L}=\bar{W}^{-1}\left(q \Lambda^{-1}\right) \bar{W} .
$$

Define evolutionary derivations $\left(\delta_{n}, \bar{\delta}_{n} \mid n>0\right)$ of $\mathcal{B}$ by the formulas

$$
\varepsilon \delta_{n} W+L_{-}^{n} W=\varepsilon \bar{\delta}_{n} W+\bar{L}_{-}^{n} W=0 .
$$

Under the embedding $\mathcal{A} \hookrightarrow \mathcal{B}$, these derivations restrict to the flows of the Toda lattice on $\mathcal{A}$.

Let $\log (L)=W \log (\Lambda) W^{-1}$, where $\log (\Lambda)$ is a formal symbol for the operator $\varepsilon \partial$. Define $\ell$ to be the difference operator

$$
\begin{aligned}
\ell & =\log (\Lambda)-\log (L)=\varepsilon(\partial W) W^{-1} \\
& =\varepsilon\left(\partial w_{k}+\sum_{j=1}^{k-1}\left(\mathrm{E}^{(k-j) / 2} \partial w_{j}\right)\left(\mathrm{E}^{-j / 2} w_{k-j}^{*}\right)\right) .
\end{aligned}
$$

The following is a result of Carlet, Dubrovin and Zhang [1]. (They work in the context of the Toda chain, so they assume that $a_{1}=\bar{a}_{1}$ and $a_{k}=0, k>2$.)

Proposition 5.1. The difference operator $\ell$ is an element of $\Phi_{-}(\mathcal{A}, q)$.

Proof. Write

$$
\ell=\sum_{k=1}^{\infty} b_{k} \Lambda^{-k} \in \Phi_{-}(\mathcal{B}, q) .
$$

We show that $b_{k} \in \mathcal{A}$ for all $k>0$, by induction on $k$.

We have

$$
\varepsilon \partial L=\varepsilon \partial\left(W \Lambda W^{-1}\right)=\varepsilon(\partial W) \Lambda W^{-1}-\varepsilon W \Lambda W^{-1}(\partial W) W^{-1}=[\ell, L],
$$

hence for each $n>0, \varepsilon \partial L^{n}=\left[\ell, L^{n}\right]$. Applying the linear map res : $\Phi_{-}(\mathcal{A}, q) \rightarrow$ $\mathcal{A}$, we obtain the equation

$$
\nabla\left([n] b_{n}+\sum_{k=1}^{n-1}[k]\left(b_{k} p_{k}(n)\right)+\mathrm{P} p_{0}(n)\right)=0 .
$$


We see that $\alpha(\partial W)=0$, and hence $\alpha(\ell)=0$. Thus, the constant of integration in (25) vanishes, and we obtain the recursion

$$
b_{n}=-\frac{1}{[n]}\left(\sum_{k=1}^{n-1}[k]\left(b_{k} p_{k}(n)\right)+\mathrm{P} p_{0}(n)\right)
$$

for the coefficients $b_{k}$, showing that they are elements of $\mathcal{A}$.

\section{$\S 6 . \quad F r a c t i o n a l$ Powers of the Lax Operator}

In this section, we study the fractional powers of the Lax operator $L$; this may be compared with the parallel construction for the KP hierarchy due to Khesin and Zakharevich [6]. The study of these fractional powers is closely related to the operator $\ell$ introduced in the last section.

Let $s$ be a complex number. The fractional power $L^{s}$ of the Lax operator $L$ is defined by means of the dressing operator:

$$
L^{s}=W \Lambda^{s} W^{-1}=\Lambda^{s}+\sum_{k=1}^{\infty} a_{k}(s) \Lambda^{s-k} \in \Phi_{-}(\mathcal{B}, q) .
$$

The coefficient $a_{k}(s)$ is given by the explicit formula

$$
a_{k}(s)=\mathrm{E}^{-s / 2} w_{k}+\sum_{j=1}^{k-1}\left(\mathrm{E}^{(k-j-s) / 2} w_{j}\right)\left(\mathrm{E}^{(s-j) / 2} w_{k-j}^{*}\right)+\mathrm{E}^{s / 2} w_{k}^{*} .
$$

In particular, $a_{k}(0)=0$ and $a_{k}(1)=a_{k}$. Differentiating the definition (27) of $L^{s}$ with respect to $s$ and setting $s=0$, we obtain the formula

$$
\left.\frac{d L^{s}}{d s}\right|_{s=0}=-\ell,
$$

showing that $a_{k}^{\prime}(0)=-b_{k}$. The following proposition is proved by extending this differential equation to all values $s$.

Proposition 6.1. The coefficient $a_{k, i}(s)$ in the expansion

$$
a_{k}(s)=\sum_{i=0}^{\infty} \varepsilon^{i} a_{k, i}(s)
$$

is a polynomial in s of degree $i+1$ with coefficients in the differential algebra

$$
\mathcal{A} \otimes_{\mathbb{Q}_{\varepsilon}} \mathbb{Q} \cong \mathbb{Q}\left\{q, a_{k} \mid k>0\right\} /(q-\bar{q}) .
$$


Proof. By its definition, the fractional power $L^{s}$ satisfies the differential equation

$$
\frac{d L^{s}}{d s}=-\frac{1}{2}\left(L^{s} \ell+\ell L^{s}\right)
$$

Taking the coefficient of $\Lambda^{s-k}$ on both sides, we obtain the differential equation

$$
\frac{d a_{k}(s)}{d s}=-\frac{1}{2} \sum_{j=1}^{k-1}\left(\mathrm{E}^{(s-j) / 2} b_{k-j} \mathrm{E}^{(k-j) / 2} a_{j}(s)+\mathrm{E}^{(j-s) / 2} b_{k-j} \mathrm{E}^{(j-k) / 2} a_{j}(s)\right),
$$

where we interpret $a_{0}(s)$ as 1 . By an application of Proposition 5.1, the result follows.

\section{$\S 7 . \quad$ Perturbation Theory for $\ell$}

Let $\Omega(\mathcal{A})$ be the vector space of Kähler differentials of the commutative $\mathbb{Q}_{\varepsilon}$-algebra $\mathcal{A}$; this is a free module over $\mathcal{A}$ with basis $\left\{d q, d a_{k}, d \bar{a}_{k} \mid k>0\right\}$. The differential $d: \mathcal{A} \rightarrow \Omega(\mathcal{A})$ extends to a morphism

$$
d: \Phi_{-}(\mathcal{A}, q) \rightarrow \Phi_{-}(\mathcal{A}, q) \otimes_{\mathcal{A}} \Omega(\mathcal{A}) .
$$

We now calculate the differentials $d L^{s}$ and $d \ell$ in terms of

$$
d L=\sum_{k=1}^{\infty} d a_{k} \Lambda^{-k+1}
$$

A basic formula of perturbation theory (Kumar [7]) says that for $f(z)$ an analytic function of $z$,

$$
d f(L)=\sum_{k=0}^{\infty} \frac{(-1)^{k}}{(k+1) !} \operatorname{ad}(L)^{k}\left(f^{(k+1)}(L) d L\right) .
$$

For $f(z)=z^{s}$, this becomes

$$
d L^{s}=\sum_{k=0}^{\infty}(-1)^{k}\left(\begin{array}{c}
s \\
k+1
\end{array}\right) \operatorname{ad}(L)^{k}\left(L^{s-k-1} d L\right) .
$$

For completeness, we will now prove this formula directly in the context in which we need it. 
For $s$ a natural number $n$, the right-hand side of (29) is a finite sum, and the formula is then easily proved by induction on $n$ : we have

$$
\begin{aligned}
d\left(L^{n+1}\right) & =d L^{n} \cdot L+L^{n} \cdot d L=\sum_{k=0}^{n-1}(-1)^{k}\left(\begin{array}{c}
n \\
k+1
\end{array}\right) \operatorname{ad}(L)^{k}\left(L^{n-k-1} d L\right) \cdot L+L^{n} \cdot d L \\
& =\sum_{k=0}^{n-1}(-1)^{k}\left(\begin{array}{c}
n \\
k+1
\end{array}\right)\left(\operatorname{ad}(L)^{k}\left(L^{n-k} d L\right)-\operatorname{ad}(L)^{k+1}\left(L^{n-k-1} d L\right)\right)+L^{n} \cdot d L \\
& \left.=\sum_{k=0}^{n}(-1)^{k}\left(\begin{array}{c}
n \\
k
\end{array}\right)+\left(\begin{array}{c}
n \\
k+1
\end{array}\right)\right) \operatorname{ad}(L)^{k}\left(L^{n-k} d L\right) \\
& =\sum_{k=0}^{n}(-1)^{k}\left(\begin{array}{c}
n+1 \\
k+1
\end{array}\right) \operatorname{ad}(L)^{k}\left(L^{n-k} d L\right) .
\end{aligned}
$$

By analytic continuation, (29) holds for all values of $s$. Indeed, the righthand side is convergent in the $\varepsilon$-adic topology, since the operation $\operatorname{ad}(L)$ may be split into two terms: $\operatorname{ad}\left(\Lambda+a_{1}\right)=O(\varepsilon)$, and

$$
\sum_{k=2}^{\infty} \operatorname{ad}\left(a_{k} \Lambda^{-k+1}\right)=O\left(\Lambda^{-1}\right) .
$$

It only remains to observe that by Proposition 6.1, the coefficient of $\varepsilon^{i}$ in $d a_{k, i}(s)$ is polynomial in $s$.

It is now straightforward to calculate $d \ell$ : taking the derivative of $(29)$ with respect to $s$ and setting $s=0$, we see that

$$
d \ell=-\sum_{k=0}^{\infty} \frac{1}{k+1} \operatorname{ad}(L)^{k}\left(L^{-k-1} d L\right) .
$$

Theorem 7.1. $\quad$ The constraint (15) defining the equivariant Toda lattice is equivalent to the identity

$$
K=L+\nu \ell-\sum_{k=1}^{\infty} \frac{z_{k}}{k} L^{-k} .
$$

The vanishing of the constants $z_{k}$ is equivalent to the constraint

$$
\left(\delta_{1}-\bar{\delta}_{1}\right) W=\nu \partial W,
$$

or equivalently, the equation $\left(\delta_{1}-\bar{\delta}_{1}\right)=\nu \partial$ on the differential algebra $\mathcal{B}$.

Proof. Written in terms of $\ell,(13)$ becomes

$$
[K-\nu \ell, L]=0 .
$$


This is equivalent to the statement that

$$
K-\nu \ell \in \mathbb{Q}_{\varepsilon, \nu}\left(\left(L^{-1}\right)\right) .
$$

It is not hard to see that for some constants $\zeta_{k}$,

$$
K-\nu \ell-L=\sum_{k=1}^{\infty} \zeta_{k} L^{-k} \in \mathbb{Q}_{\varepsilon, \nu} \llbracket L^{-1} \rrbracket ;
$$

the constant term vanishes since, by definition, $\operatorname{res}(K)$ and $\operatorname{res}(L)$ equal $v$, while $\operatorname{res}(\ell)=0$.

It remains to identify the constants $\zeta_{k}$. If $\delta$ is an evolutionary derivation of the differential algebra $\widetilde{\mathcal{A}},(30)$ implies that

$$
\delta \ell=-\sum_{k=0}^{\infty} \frac{1}{k+1} \operatorname{ad}(L)^{k}\left(L^{-k-1} \delta L\right) .
$$

In particular, since $L$ commutes with $e(L)$, we see that $e(\ell)=-L^{-1} e(L)$. Likewise, $e\left(L^{-k}\right)=-k L^{-k-1}$. Applying the derivation $e$ to both sides of (33), we see that

$$
1=e(K)=e(L)\left(1-\nu L^{-1}-\sum_{k=1}^{\infty} k \zeta_{k} L^{-k-1}\right) .
$$

It follows from Theorem 3.2 that $\zeta_{k}=-z_{k} / k$.

We have

$$
\begin{aligned}
(K-L-\nu \ell) W & =\left(L_{+}+\bar{L}_{-}\right) W-L W-\varepsilon \nu \partial W \\
& =-L_{-} W+\bar{L}_{-} W-\varepsilon \nu \partial W=\varepsilon\left(\delta_{1}-\bar{\delta}_{1}-\nu \partial\right) W
\end{aligned}
$$

Thus, the vanishing of the constants $z_{k}$ in (31) is equivalent to the constraint (32).

Theorem 7.1 implies that the equivariant Gromov-Witten invariants of $\mathbb{C P}^{1}$ are described by the equivariant Toda lattice with $z_{k}=0, k>0$. By the work of Okounkov and Pandharipande [9], the equivariant Gromov-Witten invariants of $\mathbb{C P}^{1}$ are associated with a $\tau$-function of the Toda lattice which satisfies $\left(\delta_{1}-\bar{\delta}_{1}\right) \tau=\nu \partial \tau$. The dressing operator $W$ corresponding to this $\tau$-function is given by the formula

$$
W=\tau^{-1} \exp \left(-\sum_{n=1}^{\infty} \frac{\delta_{n}}{n \Lambda^{n}}\right) \tau
$$


it follows that $W$ satisfies the equation $\left(\delta_{1}-\bar{\delta}_{1}\right) W=\nu \partial W$. Thus, the Lax operator $\mathbb{L}$ defined by the recursion (19) governs the equivariant Gromov-Witten invariants of $\mathbb{C P}^{1}$.

\section{$\S 8 . \quad H a m i l t o n i a n$ Structure}

In this section, we use Theorem 7.1 to show that the equivariant Toda lattice has a Hamiltonian structure.

Denote by $\mathcal{R}$ the quotient $\widetilde{\mathcal{A}} / \partial \widetilde{\mathcal{A}}$, and denote by $f \mapsto \int f d x$ the quotient map from $\widetilde{\mathcal{A}}$ to $\mathcal{R}$. The idea which this notation is intended to represent is that an element of $\widetilde{\mathcal{A}}$ is a density $f$, whose associated functional $\int f d x$ is obtained by integration with respect to the space variable $x$. In particular, $\int f d x$ vanishes on densities $f=\nabla g$.

Denote by Res the trace on $\Phi_{-}(\widetilde{\mathcal{A}}, q)$ with values in $\mathcal{R}$ given by the formula

$$
\operatorname{Res}(f)=\int \operatorname{res}(f) d x
$$

Clearly, this map vanishes on total derivatives; it also vanishes on commutators, by the formula

$$
\operatorname{Res}\left[\sum_{i} a_{i} \Lambda^{i}, \sum_{j} b_{j} \Lambda^{j}\right]=\nabla \sum_{k}[k]\left(a_{k} b_{-k}\right) .
$$

There is a unique linear map

$$
\operatorname{Res}: \Phi_{-}(\widetilde{\mathcal{A}}, q) \otimes_{\widetilde{\mathcal{A}}} \Omega(\widetilde{\mathcal{A}}) \rightarrow \Omega(\widetilde{\mathcal{A}}) / \partial \Omega(\widetilde{\mathcal{A}})
$$

such that $d \operatorname{Res}(A)=\operatorname{Res}(d A)$.

Associated to the equivariant Toda lattice, we have the basic sequence of functionals

$$
h_{n}=\frac{1}{n+1} \operatorname{Res}\left(L^{n+1}\right), \quad n \geq 0,
$$

with differentials $d h_{n}=\operatorname{Res}\left(L^{n} d L\right)$. In working with $h_{n}$, the following lemma is convenient.

\section{Lemma 8.1.}

$$
p_{0}(n+1)=\sum_{k=0}^{n}[k+1]\left(a_{k+1} p_{k}(n)\right)
$$


Proof. Applying the operator res to the equations $L^{n+1}=L \cdot L^{n}$ and $L^{n+1}=L^{n} \cdot L$, we see that

$$
\begin{aligned}
& p_{0}(n+1)=\mathrm{E}^{1 / 2} p_{-1}(n)+\sum_{k=0}^{\infty} \mathrm{E}^{-k / 2}\left(a_{k+1} p_{k}(n)\right), \\
& p_{0}(n+1)=\mathrm{E}^{-1 / 2} p_{-1}(n)+\sum_{k=0}^{\infty} \mathrm{E}^{k / 2}\left(a_{k+1} p_{k}(n)\right) .
\end{aligned}
$$

Taking $E^{1 / 2}$ times the second of these equations minus $E^{-1 / 2}$ times the first, we see that

$$
\nabla p_{0}(n+1)=\nabla \sum_{k=0}^{n}[k+1]\left(a_{k+1} p_{k}(n)\right)
$$

and hence, that

$$
p_{0}(n+1)=\sum_{k=0}^{n}[k+1]\left(a_{k+1} p_{k}(n)\right)+\alpha\left(p_{0}(n+1)\right) .
$$

This proves the lemma, since $\alpha\left(p_{0}(n+1)\right)=0$.

\section{Corollary 8.1.}

$$
h_{n}=\sum_{k=0}^{n} \frac{k+1}{n+1} \int\left(a_{k+1} p_{k}(n)\right) d x .
$$

For example, using the formulas (16) and (17) for $a_{2}$ and $a_{3}$, we see that

$$
\begin{aligned}
h_{0} & =\int v d x, \\
h_{1} & =\int\left(\frac{1}{2} v^{2}+a_{2}\right) d x=\int\left(\frac{1}{2} v^{2}+q+\nu v+z_{1}\right) d x, \\
h_{2} & =\int\left(\frac{1}{3} v p_{0}(2)+\frac{2}{3}\left(a_{2} p_{1}(2)\right)+a_{3}\right) d x \\
& =\int\left(\frac{1}{3} v^{3}+v[2] q+\nu\left(\frac{1}{2} v^{2}+q+\frac{1}{2} v[2] \mathrm{P} v\right)+\nu^{2} v+z_{1} v+\frac{1}{2} z_{2}\right) d x .
\end{aligned}
$$

Proposition 8.1. We have $\operatorname{Res}\left(L^{n} d K\right)=d H_{n}$, where

$$
H_{n}=h_{n}-\nu h_{n-1}+\sum_{k=1}^{n-1} z_{k} h_{n-k-1} \text {. }
$$

Proof. From (31), (29) and (30), we see that

$$
\begin{aligned}
d K & =d L+\nu d \ell-\sum_{j=1}^{\infty} \frac{z_{j}}{j} d L^{-j} \\
& =d L+\sum_{k=0}^{\infty}(k+1)^{-1} \operatorname{ad}(L)^{k}\left(\left(-\nu+\sum_{j=1}^{\infty}\left(\begin{array}{c}
j+k \\
k
\end{array}\right) z_{j} L^{-j}\right) L^{-k-1} d L\right) .
\end{aligned}
$$


Multiplying by $L^{n}$ and applying Res, all of the terms with $k>0$ drop out, and we obtain

$$
\operatorname{Res}\left(L^{n} d K\right)=\operatorname{Res}\left(\left(L-\nu+\sum_{j=1}^{\infty} z_{j} L^{-j}\right) L^{n-1} d L\right)
$$

which equals $d H_{n}$.

Let $\delta_{v}$ and $\delta_{u}$ be the variational derivatives with respect to $v$ and $u=$ $\log (q)$.

Corollary 8.2. We have $\delta_{v} H_{n}=p_{0}(n), \delta_{u} H_{n}=q p_{1}(n), \delta_{v} \bar{H}_{n}=\bar{p}_{0}(n)$ and $\delta_{u} \bar{H}_{n}=q \bar{p}_{1}(n)-\nu \mathrm{P} \bar{p}_{0}(n)$.

Proof. The formulas for $\delta_{v} H_{n}$ and $\delta_{u} H_{n}$ follow since $d K=d v+q d u \Lambda^{-1}$. The formulas for $\delta_{v} \bar{H}_{n}$ and $\delta_{u} \bar{H}_{n}$ now follow by taking conjugates, bearing in mind that $\bar{v}=v-\nu \mathrm{P} u$.

For example, we have $H_{0}=h_{0}=\int v d x$,

$$
\begin{aligned}
& H_{1}=h_{1}-\nu h_{0}=\int\left(\frac{1}{2} v^{2}+q+z_{1}\right) d x, \quad \text { and } \\
& H_{2}=h_{2}-\nu h_{1}+z_{1} h_{0}=\int\left(\frac{1}{3} v^{3}+v[2] q+\frac{1}{2} \nu v[2] \mathrm{P} v+2 z_{1} v-\nu z_{1}+\frac{1}{2} z_{2}\right) d x .
\end{aligned}
$$

It is now easy to show that the equivariant Toda lattice is Hamiltonian. Applying res to the equation $\left[K, L^{n}\right]=\nu \partial L^{n}$, we see that

$$
\nabla p_{-1}(n)=\nabla\left(q p_{1}(n)\right)+\nu \partial p_{0}(n) .
$$

It follows that $\delta_{n} v=\nabla p_{-1}(n)=\nabla\left(q p_{1}(n)\right)+\nu \partial p_{0}(n)$. In conjunction with the formula $\delta_{n} u=\nabla p_{0}(n)$, we conclude that $\delta_{n}\left[\begin{array}{l}v \\ u\end{array}\right]=\mathcal{H}\left[\begin{array}{ll}\delta_{v} H_{n} \\ \delta_{u} H_{n}\end{array}\right]$, where $\mathcal{H}$ is the Hamiltonian operator

$$
\mathcal{H}=\left[\begin{array}{cc}
\nu \partial & \nabla \\
\nabla & 0
\end{array}\right]
$$

Since $\bar{\delta}_{n} v=\nabla\left(q \bar{p}_{1}(n)\right)$ and $\bar{\delta}_{n} u=\nabla \bar{p}_{0}(n)$, we also conclude that $\bar{\delta}_{n}\left[\begin{array}{l}v \\ u\end{array}\right]=$ $\mathcal{H}\left[\begin{array}{l}\delta_{v} \bar{H}_{n} \\ \delta_{u} \bar{H}_{n}\end{array}\right]$. In other words, the equivariant Toda lattice is Hamiltonian with respect to the Hamiltonian structure

$$
\{v(x), v(y)\}=\nu \partial \delta(x-y), \quad\{v(x), u(y)\}=\nabla_{x} \delta(x-y), \quad\{u(x), u(y)\}=0 .
$$

It was proved by Getzler [5] and Zhang [14] that the flows $\partial_{k, P}$ in the Toda conjecture of Eguchi and Yang are Hamiltonian. Taking the limit $\nu \rightarrow 0$, we 
obtain the explicit formulas for these Hamiltonians due to Carlet, Dubrovin and Zhang [1]. By (6), the descendent flow $\partial_{k, P}$ has Hamiltonian

$$
\lim _{\nu \rightarrow 0}\left(\frac{H_{k+1}-\bar{H}_{k+1}}{\nu(k+1) !}-\frac{c_{k}\left(H_{k}+\bar{H}_{k}\right)}{k !}\right) .
$$

Let $\ell_{0}$ equal the limit as $\nu \rightarrow 0$ of $\ell$. Since $L=K-\nu \ell=K-\nu \ell_{0}+O\left(\nu^{2}\right)$ and

$$
\bar{L}=\bar{K}+\nu \bar{\ell}=K+\nu\left(\ell_{0}-\mathrm{P} u\right)+O\left(\nu^{2}\right),
$$

we have

$$
\begin{aligned}
\nu^{-1}\left(H_{k+1}-\bar{H}_{k+1}\right) & =\nu^{-1} \frac{1}{k+2} \operatorname{Res}\left(L^{k+2}-\bar{L}^{k+2}\right)-\frac{1}{k+1} \operatorname{Res}\left(L^{k+1}-\bar{L}^{k+1}\right) \\
& =\operatorname{Res}\left(K^{k+1}\left(\mathrm{P} u-2 \ell_{0}\right)\right) .
\end{aligned}
$$

It follows that $\partial_{k, P}$ has Hamiltonian $\frac{1}{(k+1) !} \operatorname{Res}\left(K^{k+1}\left(\mathrm{P} u-2 \ell_{0}-2 c_{k}\right)\right)$.

\section{Appendix. Another Formulation of the Equivariant Toda Conjecture}

In this appendix, we prove that $(5 \mathrm{a})$ is equivalent to

$$
\delta_{n}=n \sum_{k=1}^{n} \nu^{k-1}\left[\begin{array}{l}
n \\
k
\end{array}\right] \partial_{n-k} .
$$

For example, $\frac{1}{2} \delta_{2}=\partial_{1}+\nu \partial_{0}$ and $\frac{1}{6} \delta_{3}=\partial_{2}+3 \nu \partial_{1}+2 \nu^{2} \partial_{0}$. A similar proof, which we omit, shows that $(5 \mathrm{~b})$ is equivalent to

$$
\bar{\delta}_{n}=n \sum_{k=1}^{n}(-\nu)^{k-1}\left[\begin{array}{l}
n \\
k
\end{array}\right] \bar{\partial}_{n-k} .
$$

Equation (5a) may be restated as saying that

$$
\partial_{k}=\sum_{n=1}^{k+1}(-\nu)^{k-n+1} h_{k-n+1}\left(1, \frac{1}{2}, \ldots, \frac{1}{n}\right) \frac{\delta_{n}}{n !},
$$

where $h_{\ell}$ is the complete symmetric polynomial of degree $\ell$. We wish to prove that

$$
\frac{\delta_{n}}{n !}=\sum_{\ell=0}^{n-1} \nu^{n-\ell-1} e_{n-\ell-1}\left(1, \frac{1}{2}, \ldots, \frac{1}{n-1}\right) \partial_{\ell}
$$

where $e_{\ell}$ is the elementary symmetric polynomial of degree $\ell$. In other words, we wish to prove that

$$
\sum_{\ell=0}^{n-1} \nu^{n-\ell-1}(-\nu)^{\ell-m+1} e_{n-\ell-1}\left(1, \frac{1}{2}, \ldots, \frac{1}{n-1}\right) h_{\ell-m+1}\left(1, \frac{1}{2}, \ldots, \frac{1}{m}\right)=\delta_{n, m} .
$$


This is clearly true if $n \leq m$; thus, we have only to prove that the left-hand side vanishes when $n>m$. In this case, it equals $\nu^{n-m}$ times the coefficient of $\nu^{n-m}$ in the generating function

$$
\prod_{j=1}^{n-1}(1+j \nu) \cdot \prod_{j=1}^{m}(1+j \nu)^{-1}=\prod_{j=m+1}^{n-1}(1+j \nu),
$$

which is a polynomial of degree $n-m-1$; hence, the coefficient in question vanishes.

\section{Acknowledgements}

In writing this paper, I profited greatly from discussions with T. Eguchi and R. Pandharipande (at the Institute for Theoretical Physics of the University of California, Santa Barbara) and with B. Dubrovin and Y. Zhang (at the Institute for Advanced Study, Princeton, and the Max-Planck-Institut für Mathematik, Bonn). I thank these institutes for their hospitality and support.

\section{References}

[1] Carlet, G., Dubrovin, B. and Zhang, Y., The extended Toda hierarchy. nlin.SI/0306060

[2] Dijkgraaf, R. and Witten, E., Mean field theory, topological field theory, and multimatrix models, Nucl. Phys. B, 342 (1990), 486-522.

[3] Eguchi, T. and Yang, S.-K., The topological $\mathbb{C P}^{1}$ model and the large- $N$ matrix integral, Modern Phys. Lett. A, 9 (1994), 2893-2902. hep-th/9407134

[4] Eguchi, T., Hori, K. and Yang, S.-K., Topological $\sigma$-models and large- $N$ matrix integral, Internat. J. Modern Phys. A, 10 (1995), 4203-4224. hep-th/9503017

[5] Getzler, E., The Toda conjecture, in: Fukaya, K. et al. eds., Symplectic geometry and mirror symmetry (KIAS, Seoul, 2000), World Scientific, Singapore, 2001, 51-79. math. AG/0108108

[6] Khesin, B. and Zakharevich, I., Poisson-Lie group of pseudodifferential symbols and fractional KP-KdV hierarchies, C. R. Acad. Sci. Paris Sér. I Math., 316 (1993), 621626. hep-th/9311125

[7] Kumar, K., Expansion of a function of noncommuting operators, J. Math. Phys., 6 (1965), 1923-1927.

[8] Kupershmidt, B. A., Discrete Lax equations and differential-difference calculus, Astérisque, 123 (1985).

[9] Okounkov, A. and Pandharipande, R., The equivariant Gromov-Witten theory of $\mathbf{P}^{1}$. math.AG/0207233

[10] Pandharipande, R., The Toda equations and the Gromov-Witten theory of the Riemann sphere, Lett. Math. Phys., 53 (2000), 59-74. math. AG/9912166

[11] , private communication (2000).

[12] Takasaki, K. and Takebe, T., Quasi-classical limit of Toda hierarchy and $W$-infinity symmetries, Lett. Math. Phys., 28 (1993), 165-176.

[13] Ueno, K. and Takasaki, K., Toda lattice hierarchy, in: Group representations and systems of differential equations (Tokyo, 1982), Adv. Stud. Pure Math., 4 North-Holland, Amsterdam, 1984, 1-95.

[14] Zhang, Y., On the $\mathbb{C P}^{1}$ topological sigma model and the Toda lattice hierarchy, J. Geom. Phys., 40 (2002), 215-232. 